\title{
TLNC-UC.147, A NOVEL LONG RNA (InCRNA) FROM AN ULTRACONSERVED REGION AS POTENTIAL BIOMARKER IN LUMINAL A BREAST CANCER
}

Erika Pereira Zambalde, Ana Carolina Rodrigues', Rubens Silveira Lima, Enilze Maria Souza Fonseca Ribeiro, Jaqueline Carvalho Oliveira ${ }^{1}$

${ }^{1}$ Universidade Federal do Paraná - Curitiba (PR), Brazil.

Introduction: Long RNAs are non-coding RNAs with more than 200 nucleotides in length, with essential regulatory roles in several biological processes, including in breast cancer (BC). The human genome contains 481 ultraconserved regions, which are genomic stretches of over 200 base pairs conserved among humans, rats, and mice. Most of these regions are transcriptionally active (T-UCRs), and several are differentially expressed in tumors. Some T-UCRs have been functionally characterized, but few have been associated with BC. Objectives: In this study, we aimed to expand the knowledge of T-UCRs in BC and characterize the lnc-uc.147, a long RNA transcribed from an ultraconserved region. Methods: We evaluated the expression level of 481 T-UCRs and their association with clinical parameters from TCGA data. For confirmation, 102 Brazilian BC samples were analyzed by RT-qPCR. Cytosolic and nuclear cell fractions and RT-qPCRs were done to determine the cell compartment of the transcript. Northern blotting and RACE were performed to determine the sequence and precise size of Inc-uc.147. Using two luminal cell lines (CAMA and BT474), a siRNA-based approach was applied to investigate the effects of lnc-uc.147 knockdown in cell viability, colony formation, and apoptosis level. To understand the interactions of lnc-uc.147 and proteins, we performed a pull-down assay. Results: Using TCGA (The Cancer Genome Atlas) data, we found 302 T-UCRs related to clinical features in BC: 43\% were associated with molecular subtypes, 36\% with estrogen-receptor positivity, $17 \%$ with HER2 expression, $12 \%$ with stage, and $10 \%$ with overall survival. We found that uc.147 is highly expressed in luminal A and B patients, which was also confirmed in Brazilian samples. For luminal A, a subtype usually associated with better prognosis, high uc.147 expression was associated with a poor prognosis and suggested as an independent prognostic factor. The lncRNA from uc.147 (lnc-uc.147) is in the nucleus. Northern blotting results show that uc. 147 is a 2,8 kb monoexonic transcript. The silencing of uc. 147 increases apoptosis, arrests the cell cycle and reduces cell viability and colony formation in luminal BC cell lines. Additionally, we identified 19 proteins that interact with uc.147 through mass spectrometry. These proteins are mainly involved in cytoskeletal and centrosome organization as well as in epithelial-mesenchymal transition. Conclusions: We show herein evidence that neoplastic BC cells exhibit a unique expression profile of T-UCRs. This study characterized the lnc-uc.147, a transcript that has never been described before. Indeed, Inc-uc.147 has an oncogenic effect in the luminal BC cell line and can interact with proteins. Furthermore, uc.147 has the potential as a BC prognostic marker in luminal patients. 